\title{
A comparison between conventional throat packs and pharyngeal placement of tampons in rhinology surgeries.
}

\author{
Hasan Alansari ${ }^{1}$, amr abdullah ${ }^{1}$, Abdulrahman AlMannai ${ }^{1}$, Raneem Al shaikh ${ }^{1}$, Eman \\ Juma $^{1}$, Hiba Alreefy ${ }^{2}$, and keith johnston ${ }^{1}$ \\ ${ }^{1}$ King Hamad University Hospital \\ ${ }^{2}$ William harvey hospital
}

August 17, 2020

\begin{abstract}
Abstract Objective: the aim is to evaluate the incidence of postoperative throat pain, nausea and vomiting in patients that have been packed with either conventional gauze or pharyngeal tampons. Methods We included adult patients who were booked for a rhinology surgery that needed throat packs. They were allocated into two groups, pharyngeal tampons, and conventional ribbon gauze. They were then assessed using visual analog scales and PONV impact scale respectively in the 1st, 4th, and 24 th -hour. Results VAS results show that tampons are better than gauze, with 25 out of 43 patients using tampons having low VAS scores after 24 hours whereas 23 out of 46 patients using gauze had low VAS scores after 24 hours. The data shows that tampons are equivalent to gauze in terms of PONV. Conclusion The study shows that the use of pharyngeal tampons is associated with decreased pain scores, however, it also shows that there no difference in postoperative nausea and vomiting.
\end{abstract}

A comparison between conventional throat packs and pharyngeal placement of tampons in rhinology surgeries.

Abstract

Objective: the aim is to evaluate the incidence of postoperative throat pain, nausea and vomiting in patients that have been packed with either conventional gauze or pharyngeal tampons.

Methods We included adult patients who were booked for a rhinology surgery that needed throat packs. They were allocated into two groups, pharyngeal tampons, and conventional ribbon gauze. They were then assessed using visual analog scales and PONV impact scale respectively in the 1st, 4th, and 24th -hour.

Results VAS results show that tampons are better than gauze, with 25 out of 43 patients using tampons having low VAS scores after 24 hours whereas 23 out of 46 patients using gauze had low VAS scores after 24 hours.

The data shows that tampons are equivalent to gauze in terms of PONV.

Conclusion The study shows that the use of pharyngeal tampons is associated with decreased pain scores, however, it also shows that there no difference in postoperative nausea and vomiting.

Bullet points

1. Posterior throat packs are often-used in rhinology surgery to help in protecting the airway against aspiration and in the reduction of ponv

2. A conventional throat pack consists of gauze, but an alternative practice involves the pharyngeal placement of tampons 
3. Patient that were packed with pharyngeal tampons had a lower pain scores than the conventional throat pack group

4. that there are no differences in post-operative nausea and vomiting in both groups.

5. pharyngeal tampons are a suitable alternative -if not replacement - for conventional ribbon gauze

\section{Introduction}

The nose and para nasal sinuses are anatomically known to be highly vascular therefore a substantial amount of bleeding is expected from rhinology surgeries. Ingested blood is known to be a powerful emetic ${ }^{1,2}$, and the risk of aspirating blood into the lungs is present due to the fact that an endotracheal tube is not $100 \%$ effective in protecting the airway ${ }^{3,4}$. Post-operative nausea and vomiting (PONV) is a frequent problem that is encountered in surgical patients with a 4 to 6 -fold increase in nasal and endoscopic sinus operations ${ }^{1,4}$.

Posterior throat packs are often-used in rhinology surgery to help in protecting the airway against aspiration and in the reduction of ponv ${ }^{5}$. However, many studies concluded that posterior throat packs are associated with higher amounts of post-operative pain, nausea ${ }^{2,6,7}$, vomiting, and in some rare cases even forgotten in the patient leading to mortality ${ }^{8}$. Despite poor evidence in the literature, the placement of posterior throat packs is commonly practiced by surgeons and anesthetists because the possible risk of aspiration is worrisome ${ }^{9}$.

A conventional throat pack consists of gauze, but an alternative practice involves the pharyngeal placement of tampons. The objective of this study is to evaluate and compare the post-operative throat pain, nausea, and vomiting in patients that have been packed with either conventional gauze throat packs or pharyngeal tampons.

\section{Material and methods}

In this prospective clinical trial, all surgeries were done by a single surgeon as to decrease variability in the management of each patient. Patients were divided into two groups, one that received the conventional gauze throat packs and those that received pharyngeal tampons (figure 1.1 and figure 1.2). The allocation of patients was done on the day of the surgery dependant on the first patient that was admitted in the morning, the allocation of the first patient was decided by coin toss, if the first patient was kept in the pharyngeal tampon group the subsequent patient was kept in the conventional guaze throat pack group and it alternated till the end of the list. This allowed for randomisation and equal distribution among the 2 groups.

Our study population included all adult patients that underwent rhinology procedures that required the use of a throat pack between January of 2018 to January of 2019., Minors below the age of 18 and rhinology procedures that required no throat pack were excluded from the study. The Institutional review board provided ethical approval for approved the study, and signed informed consent was obtained from all patients.

Post-operative pain was assessed using the visual analogue scale ${ }^{10}$, (figure 1.3) patients were asked on a scale of 1 to 10 on the amount of pain they were experiencing in various post op periods, where 1 was the lowest amount of pain and 10 was the highest amount of pain. Patients were seen at 1 hour and on the $4^{\text {th }}$ hour post operatively and were called via phone 24 hours later to assess the amount of pain they were experiencing. All data collected was recorded in an excel sheet.

Post-operative nausea was assessed using the PONV impact scale ${ }^{11}$ (figure 1.4). It includes 2 questions, one of which is whether the patient had experienced any dry retching or vomiting, and the second one being if they experienced nausea. A final score (PONV Impact scale) was calculated using the responses to the previous two questions. A PONV Impact Score of [?]5 defines clinically important PONV. Patients were seen at 1 hour and on the $4^{\text {th }}$ hour post operatively and were called via phone 24 hours later, to assess the amount of nausea and vomiting they were experiencing. All data collected was recorded in an excel sheet.

$\mathrm{T}$ Test and levenes test was done on the date and All data was analyzed using SPSS Statistics software version 25 . 


\section{Results}

The total number of patients included in this study was 89, with $46(51.6 \%)$ being placed in the pharyngeal tampon group and $43(49.4 \%)$ in the conventional throat pack group. The average age of the patients was 32.8 years (ranging from 18-72). Out of the 89 patients, 49 patients were male, and 40 patients were female, with a ratio of $1.225: 1$.

|Eighty-nine procedures were performed in this year, and of those Functional endoscopic sinus surgery comprised of 27, septoplasties 25, rhinoplasties 23, septoplasty/turbinoplasty 8 and turbinoplasties 6 .

The mean PONV score at 1 hour for the tampon group was 0.28 and the mean score for the gauze group was 0.54. Mean difference between the groups was 0.264. The mean PONV score at 4 hours for the tampon group was 0.37 , and the mean score for the gauze group was 0.41 . Mean difference between the groups was 0.41. The mean PONV score at 24 hours for the tampon group was 0.21 and the mean score for the gauze group was 0.26 . Mean difference between the groups was $0.52{ }^{\text {table }} 1.1$ and 1.2

The mean VAS score at 1 hour for the tampon group was 1.51 and the mean score for the gauze group was 2.28. Mean difference between the groups was 0.771. The mean VAS score at 4 hours for the tampon group was 1.28, and the mean score for the gauze group was 2.85. Mean difference between the groups was 1.569. The mean VAS score at 24 hours for the tampon group was 2.00, and the mean score for the gauze group was 2.17. Mean difference between the groups was 0.174 . table 2.1 and 2.2

\section{Discussion}

There is no evidence to suggest that using pharyngeal tampons reduces the mean VAS score at $1[\mathrm{t} 87=1.368$, $\mathrm{p}=0.175]$ or 24 hours [ $\mathrm{t} 87=0.310, \mathrm{p}=0.757$ ], compared to using Gauze. There is evidence to suggest that using pharyngeal tampons reduces the mean VAS score at 4 hours [t87 $=3.294, \mathrm{p}=0.001]$ compared to using gauze.

The pattern of data shows that the tampon is better than gauze in terms of VAS. 25 out of 43 patients using tampons had low VAS scores after 24 hours, whereas 23 out of 46 patients using gauze had low VAS scores after 24 hours.

There is no evidence to suggest that using pharyngeal tampons reduces the mean PONV score at $1\left[\mathrm{t}_{87}=1.389\right.$, $\mathrm{p}=0.168], 4\left[\mathrm{t}_{87}=-0.247, \mathrm{p}=0.806\right]$ and 24 hours [ $\left.\mathrm{t}_{87}=0.389, \mathrm{p}=0.698\right]$, compared to using Gauze.

The pattern of data shows that the tampon is equivalent to gauze in terms of PONV. 36 out of 43 patients using tampons had a low PONV score after 24 hours, and 39 out of 46 patients using gauze showed similar scores

There is limited research on the use of pharyngeal tampons in rhinology procedures. Marais and Prescott (1993) showed that the use pharyngeal tampons are associated with a decreased amount of post-operative throat pain, when compared to conventional gauze throat packs ${ }^{12}$. A research paper by Green et al (2017) advises against the use of.... conventional pharyngeal packs due to higher incidences of post-operative pain ${ }^{9}$. Our study shows that pharyngeal tampon placement is associated with decreased amount of pain and could be suitable alternative to conventional ribbon gauze packs.

We could not find any studies that compared the post-operative nausea and vomiting in the two different types of throat packs, however in Basha et al (2006), they established that packing the patient with conventional ribbon gauze was associated with a higher chance of post-operative nausea and vomiting immediately post$\mathrm{op}^{13}$. Piltcher et (2007) al compared $p$ ost-operative nausea and vomiting in packed and unpackedprocedures and came to the conclusion that there is no difference in throat pain or PONV ${ }^{1}$. Our study concluded that use of tampons is equivalent to gauze in terms of PONV.

Our study was dependent on subjective methods of assessment thus each patient experienced a different degree of pain and PONV. Patients around the 1-hour post -operative mark were still under theresidual effects of anesthesia and analgesia which would Heavily skew their assessment. Also, although the cases were 
done under the care of a single surgeon, the anaesthetic management was not. The method of throat pack application was not standardized for the study and as in adding an unforeseen variable to the scoring of the . Our study would have benefited from a larger population and further studies should be carried out in order to confirm our results.

\section{Conclusion}

$O$ ur study shows that the use of pharyngeal tampons is associated with decreased pain scores, however, it also shows that there are no differences in post-operative nausea and vomiting. We believe that pharyngeal tampons are a suitable alternative -if not replacement - for conventional ribbon gauze. Further studies with higher population numbers should be carried to confirm these results.

References:

1. Piltcher O, Lavinsky M, Lavinsky J, Basso PRO. Effectiveness of hypopharyngeal packing during nasal and sinus surgery in the prevention of PONV. Otolaryngol Head Neck Surg. 2007; 137:552-554.

2. Fennessy BG, Mannion S, Kinsella JB, O'Sullivan P. The benefits of hypopharyngeal packing in nasal surgery: a pilot study. Ir J Med Sci. 2011;180:181-183.

3. Seraj MA, Ankutse MM, Khna FM, Siddiqui N, Ziko AO. Tracheal soiling with blood during intranasal surgery. Middle East J Anesthesiology. 1991;11:79-89.

4. Meco B, Ozcelik M, Yildirim Guclu C, Beton S, Islamoglu Y, Turgay A et al. Does Type of Pharyngeal Packing during Sinonasal Surgery Have an Effect on PONV and Throat Pain?. Otolaryngology-Head and Neck Surgery. 2016;154(4):742-747.

5. Jaiswal V, Bedford G. Review of the use of throat packs in nasal surgery. The Journal of Laryngology \& Otology. 2009;123(07):701.

6. Korkut AY, Erkalp K, Erden V, et al. Effect of pharyngeal packing during nasal surgery on postoperative nausea and vomiting. Otolaryngol Head Neck Surg 2010;143:831-836.

7. Singh R, Rasheed M, Sarkar A, Rizvi M. Effects of different types of pharyngeal packing in patients undergoing nasal surgery: A comparative study. Anesthesia: Essays and Researches. 2015;9(2):230.

8. Tay JY, Tan WK, Chen FG, et al. Postoperative sore throat after routine oral surgery: influence of the presence of a pharyngeal pack. Br J Oral Maxillofac Surg 2002;40:60 -3.

9. Green R, Konuthula N, Sobrero M, Saini A, Parasher A, Pool C et al. Use of pharyngeal packs in functional endoscopic sinus surgery: A randomized controlled trial. The Laryngoscope. 2017;127(11):24602465 .

10. Assessment-module.yale.edu. (2020). Visual Analogue Scale| Yale Assessment Module Training . [online] Available at: https://assessment-module.yale.edu/im-palliative/visual-analogue-scale [Accessed 5 Mar. 2020].

11. Myles, P. and Wengritzky, R. (2020). Simplified postoperative nausea and vomiting impact scale for audit and post-discharge review

12. Marais J, Prescott RJ. Throat pain and pharyngeal packing: A controlled randomized double-blind comparison between gauze and tampons. Clin Otolaryngol Allied Sci. 1993;18:426-9.

13. Basha SI, McCoy E, Ullah R, Kinsella JB. The efficacy of pharyngeal packing during routine nasal surgery - a pro-spective randomised controlled study. Anaesthesia 2006;61:1161 - 5

\section{Hosted file}

tables.docx available at https://authorea.com/users/351348/articles/476002-a-comparisonbetween-conventional-throat-packs-and-pharyngeal-placement-of-tampons-in-rhinologysurgeries 

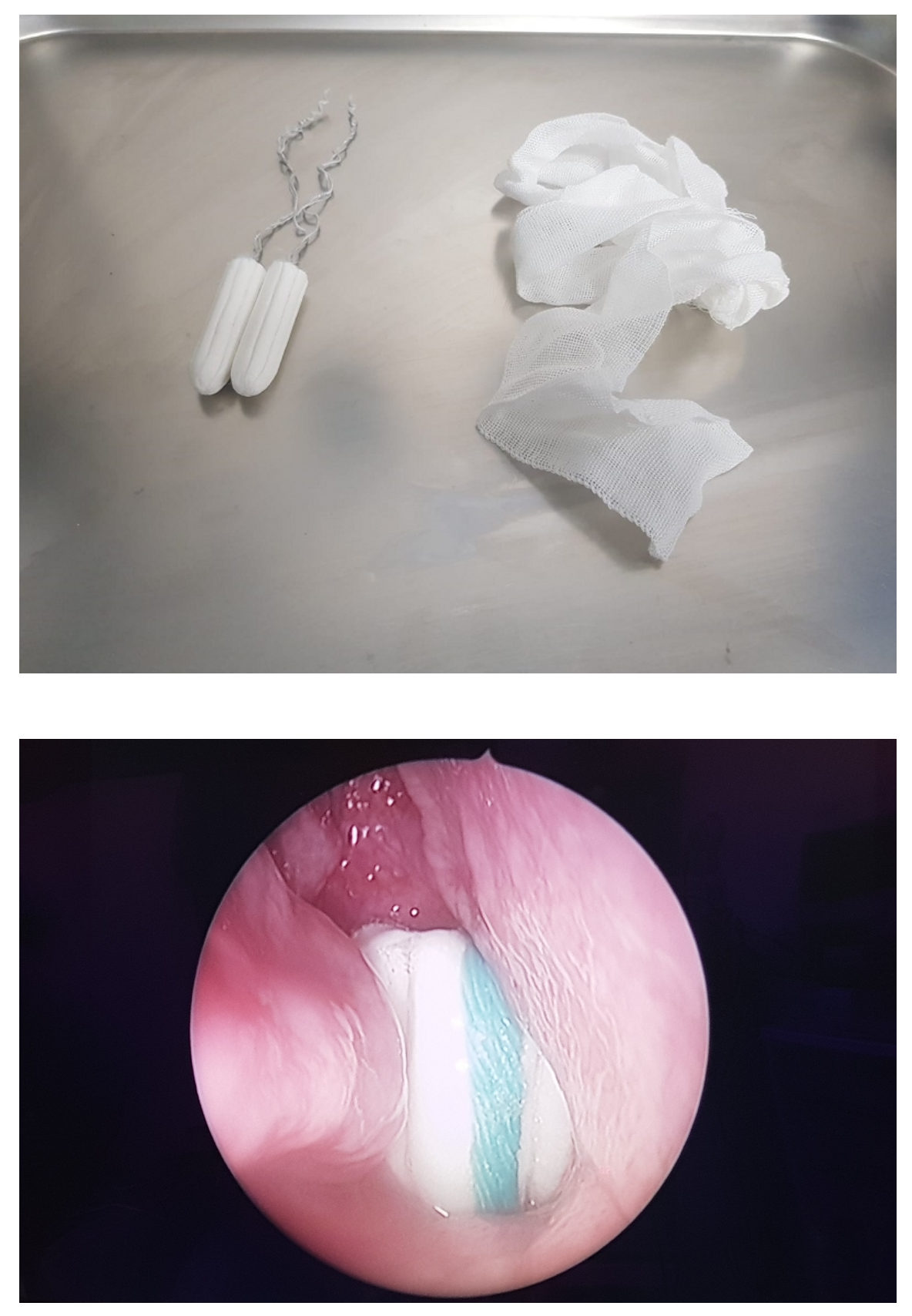


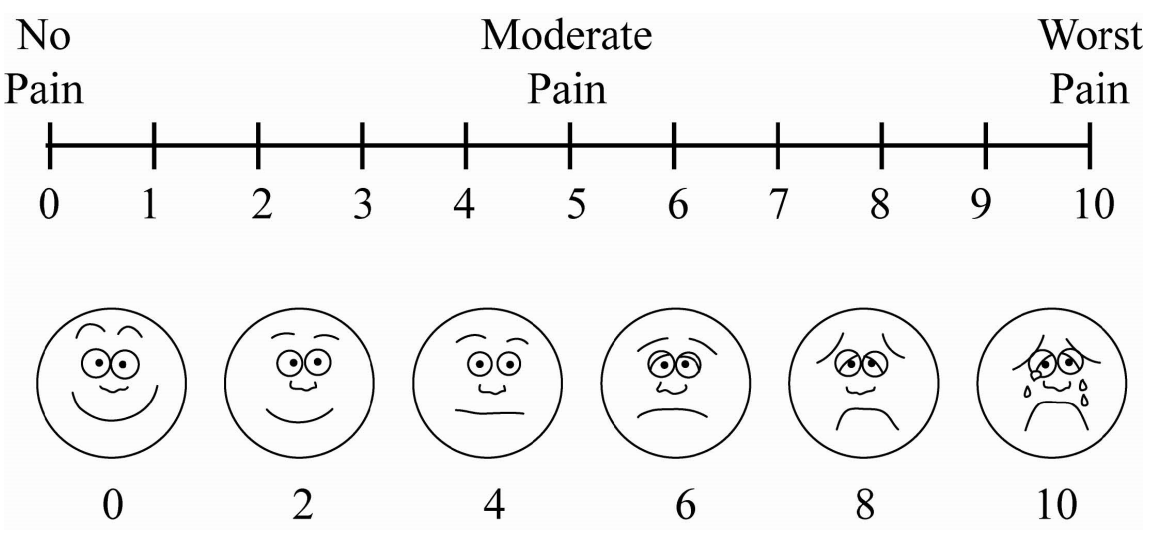

Q1. Have you vomited or had dry-retching*?

0 . No

1. Once

2. Twice

3. Three or more times

Q2. Have you experienced a feeling of nausea ("an unsettled feeling in the stomach and slight urge to vomit")? If yes, has your feeling of nausea interfered with activities of daily living, such as being able to get out of bed, being able to move about freely in bed, being able to walk normally, or eating and drinking?

0 . Not at all

1. Sometimes

2. Often or most of the time

3. All of the time.

To calculate the PONV Impact Scale score, add the numerical responses to questions 1 and 2. A PONV Impact Scale score of $\geq 5$ defines clinically important PONV.

"count distinct episodes: several vomits or retching events occurring over a short time frame, say 5 min, should be counted as one vomiting/dry-retching episode; multiple episodes require distinct time periods without vomiting/dry-retching. 